\title{
Otimização da aceitabilidade sensorial de requeijão cremoso light
}

\author{
Optimization of the sensory acceptability of light cream cheesecurd
}

\author{
Rita de Cássia dos Santos Navarro da Silva ${ }^{\mathrm{I}}$ Valéria Paula Rodrigues Minim ${ }^{{ }^{*}}$ Luiz Paulo de Lima ${ }^{\mathrm{I}}$ \\ Aline Iamin Gomide ${ }^{\mathrm{I}}$ Liliane Elen da Silva Moraes ${ }^{\mathrm{I}}$ Luis Antônio Minim ${ }^{\mathrm{I}}$
}

\section{RESUMO}

O requeijão cremoso é um tipo de queijo fundido originalmente brasileiro, fabricado em todo território nacional com algumas variações de tecnologia. Este é um dos principais tipos de queijos produzido e consumido no Brasil e apresenta como característica o elevado teor de gordura. Por outro lado, a gordura apresenta papel fundamental na aparência, sabor $e$ textura de requeijão, portanto desenvolver um produto light com qualidade sensorial semelhante à versão tradicional é um grande desafio à indústria de alimentos. Nesse contexto, o objetivo deste trabalho foi avaliar a composição química e a aceitabilidade sensorial de requeijão cremoso light, com diferentes níveis de redução de gordura, em relação à textura e impressão global. O experimento seguiu um delineamento de superfície de resposta, o Delineamento Composto Central Rotacional (DCCR) com dois fatores (gordura e umidade). A aceitabilidade sensorial foi avaliada por 100 consumidores, utilizando a escala hedônica de nove pontos. Os resultados obtidos demonstraram que os requeijões com diferentes níveis de redução de gordura apresentaram aceitabilidade sensorial semelhante quando combinados a um aumento proporcional do teor de umidade. Os produtos que apresentaram extrato seco desengordurado (ESD) de aproximadamente 19,3\%, originados de diferentes combinações entre gordura e umidade, foram os que apresentaram melhor aceitação por parte dos consumidores, demonstrando a importância do ESD na aceitabilidade sensorial.

Palavras-chave: requeijão cremoso light, gordura, umidade, aceitabilidade sensorial.

\section{ABSTRACT}

Cream cheesecurd is a type of processed cheese, originally Brazilian, produced throughout the entire Brazilian territory with some technological variations. This is one of the main types of cheese produced and consumed in Brazil and it is known for presenting an elevated fat content. On the other hand, the fat plays a fundamental role in appearance, flavor and texture of cheese, then developing a light product with sensory quality similar to the traditional version is a major challenge to food industry. In this context, the objective of this study was to evaluate the chemical composition and sensory acceptability of light cream cheesecurd with different levels of fat reduction for texture and overall impression. The experiment followed a design of response surface, the Central Composite Rotatable Design (CCRD) with two factors (fat and moisture). The sensory acceptability was evaluated by 100 consumers, by the hedonic scale of nine points. The results showed that the cheesecurds with different levels of fat reduction had similar sensory acceptability when combined with a proportional increase in moisture content. The products that presented dry defatted extract (DDE) of approximately 19.3\%, originated from different combinations of fat and moisture, were those with better acceptance by consumers, demonstrating the importance of DDE in sensory acceptance.

Key words: light cream cheesecurd, fat, moisture, sensory acceptability.

\section{INTRODUÇÃO}

Os consumidores em geral têm adquirido um maior entendimento da relação entre produtos light e a saúde, sendo que o principal motivo dessa preocupação está relacionado ao consumo de produtos com elevado teor de gordura, tais como queijos e outros derivados lácteos (ROMEIH et al., 2002). Por outro lado, os consumidores não abrem mão da qualidade sensorial

IDepartamento de Tecnologia de Alimentos, Universidade Federal de Viçosa (UFV), Avenida PH Holfs s/n, Campus Universitário, 36570-000, Viçosa, MG, Brasil. E-mail: vprm@ufv.br *Autor para correspondência. 
destes alimentos, exigindo que o produto light apresente propriedades sensoriais semelhantes ao produto tradicional (HENNELLY et al., 2006).

A gordura é um fator determinante do sabor, textura e aparência dos alimentos, portanto, desenvolver um produto com reduzido teor de gordura, mas com a mesma qualidade da versão tradicional se torna um grande desafio às indústrias de alimentos (ROMEIH et al., 2002). A redução do teor de gordura de um alimento pode ser responsável pela rejeição sensorial deste produto, particularmente devido aos defeitos na textura, sendo que, em queijos, tal defeito está associado ao concomitante aumento do extrato seco desengordurado (ESD) como consequência da redução da gordura (SHEEHAN \& GUINEE, 2004). O elevado conteúdo caseínico contido no ESD promove o fortalecimento das interações proteína-proteína, causando o enrijecimento da matriz protéica e, assim, diminuição da cremosidade do requeijão (KAHYAOGLU \& KAYA, 2003).

Diversas estratégias têm sido utilizadas para compensar os efeitos negativos da redução da gordura em queijos, incluindo adição de substitutos de gordura (RUDAN et al.1999); adição de culturas lácteas produtoras de exopolissacarídeos (PERRY et al., 1998); e/ou alterações no processamento para diminuição do teor de ESD (METZGER et al., 2001). Esses métodos apresentam diferentes graus de sucesso.

O aumento do teor de umidade em queijos light se apresenta como uma estratégia simples para redução do ESD, visando a minimizar os efeitos negativos da redução da gordura por meio da dissolução da matriz protéica. Nesse sentido, este estudo teve como objetivo avaliar a composição química e o efeito do teor de gordura e umidade na aceitabilidade sensorial de requeijão cremoso com baixo teor de gordura adicionado de concentrado protéico de soro.

\section{MATERIAL E MÉTODOS}

Para definir as formulações, foi utilizado Delineamento Composto Central Rotacional (DCCR) com dois fatores (gordura $(\mathrm{G})$ e umidade $(\mathrm{U})$ ) em dois níveis $\left(2^{2}\right)$, mais quatro pontos axiais $(2 \times 2)$ e o ponto central. O ponto central foi repetido três vezes para estimativa do erro puro, perfazendo um total de 11 ensaios (Figura 1). Os níveis de gordura em estudo foram definidos de maneira que a formulação com maior percentual de gordura atendesse à redução mínima exigida pela legislação para ser considerada light (BRASIL, 1998). Para tanto, foi fixado como referência um requeijão com 25\% de gordura (valor médio de produtos comerciais).

Os requeijões foram processados na planta piloto do Departamento de Tecnologia de Alimentos da Universidade Federal de Viçosa (UFV), utilizando massa obtida por acidificação direta do leite, conforme recomendado por ALVES et al. (2007). Para obtenção da massa básica, o leite cru desnatado foi precipitado com ácido lático $85 \%$ PA a $70^{\circ} \mathrm{C}$. A massa foi prensada e empregada juntamente com creme de leite $(40 \% \mathrm{G})$, concentrado protéico de soro (2,0\% de CPS 34 -

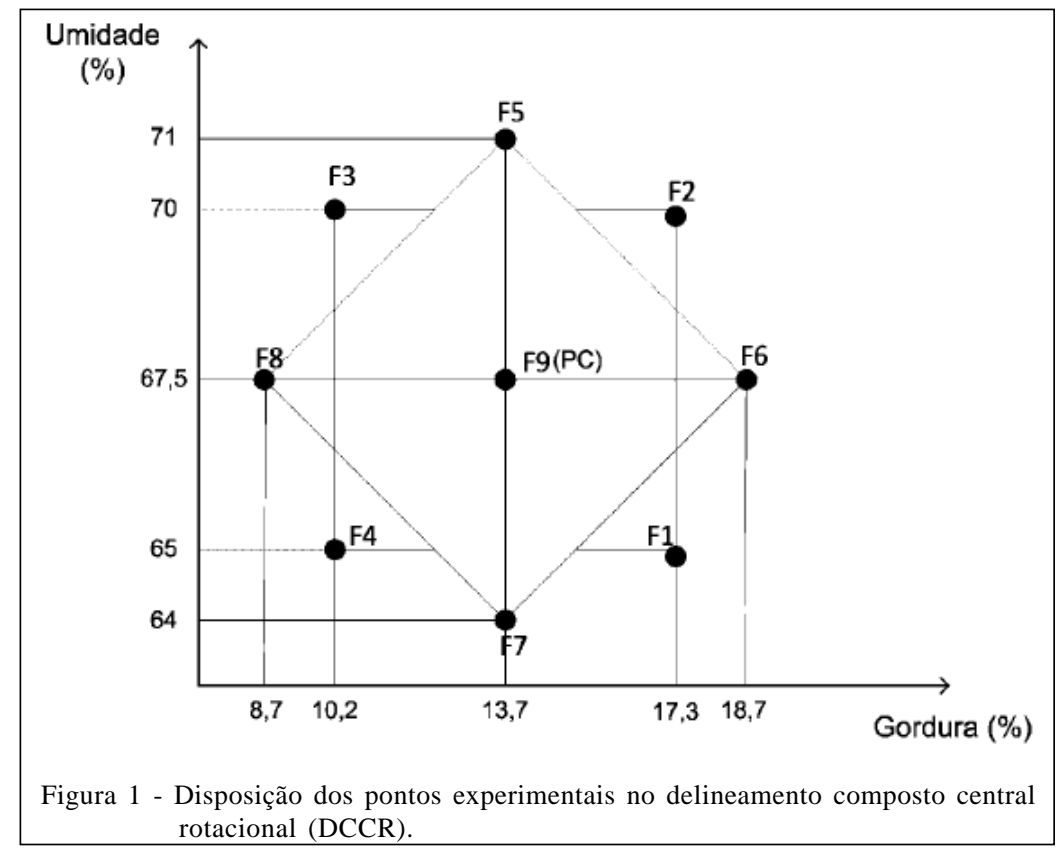

Ciência Rural, v.42, n.2, fev, 2012. 
Gemacom), $\mathrm{NaCl}(0,80 \%$ - Cisne), sal fundente (0,70\% Joha S9), água e conservantes sorbato de potássio (0,020\% - Gemacom) e nisina (0,017\% - Danisco). O processamento foi realizado em tacho aberto provido de agitador mecânico.

\section{Composição centesimal}

Foram determinados os teores de gordura, umidade, proteínas, resíduo mineral fixo e carboidratos. $\mathrm{O}$ teor de gordura foi determinado pelo método de Gerber, utilizando butirômetro específico; a umidade por meio da evaporação em estufa à temperatura de $105^{\circ} \mathrm{C}$; as proteínas pelo método de Kjeldahl e o resíduo mineral fixo (cinzas) por calcinação em forno mufla (BRASIL, 2005). Os teores de carboidratos e extrato seco desengordurado e o conteúdo calórico dos requeijões foram obtidos por meio do método indireto (BRASIL, 2005; USDA, 1963). As análises para determinação da composição centesimal dos requeijões foram realizadas em duplicata para cada repetição.

Para verificar se os teores de gordura e de umidade dos requeijões processados corresponderam aos níveis estipulados pelo delineamento experimental (DCCR), foi realizado um teste t para amostras independentes entre o valor teórico e o obtido na prática por meio da determinação da composição centesimal.

Teste com consumidor: Aceitabilidade sensorial

Os testes sensoriais foram realizados no Laboratório de Propriedades Tecnológicas e Sensoriais dos Alimentos do Departamento de Tecnologia de Alimentos. Foram recrutados, no Campus da Universidade Federal de Viçosa (UFV), 100 consumidores de requeijão. As formulações de requeijão foram servidas aos consumidores de forma monádica e aleatória, em copos descartáveis contendo aproximadamente $10 \mathrm{~g}$ do produto. Utilizou-se a escala hedônica de nove pontos, variando de "gostei extremamente” (escore 9) a “desgostei extremamente” (escore 1), para os julgadores expressarem a aceitação em relação à textura e impressão global dos produtos (MINIM, 2010). A análise de resultados foi realizada por meio do Mapa de Preferência Interno e Superfície de Resposta, a fim de verificar o comportamento da aceitabilidade sensorial em função do teor de gordura e de umidade.

\section{RESULTADOS E DISCUSSÃO}

Análises físico-químicas: composição centesimal dos requeijões

A composição centesimal das formulações (F1 a F9) de requeijão cremoso light estão apresentadas na tabela 1. Os níveis teóricos e experimentais de gordura e umidade dos requeijões não apresentaram diferença significativa pelo teste $\mathrm{t}$, apresentado p-valor de 0,8670 e 0,7887 , respectivamente. Isso indica que as formulações processadas atenderam aos requisitos estipulados pelo delineamento.

As diferentes combinações entre gordura e umidade produziram requeijões com teor de extrato seco desengordurado diferenciado. Assim, a redução do teor de gordura e umidade acarretou um aumento do teor de proteína (caseína) nos requeijões. Resultado semelhante foi encontrado por SOARES et al. (2002) e por CUNHA et al. (2006) na determinação da composição centesimal de queijos com baixo teor de gordura.

Esta relação foi observada nas formulações estudadas, em que os requeijões F2 e F3 apresentaram

Tabela 1 - Composição centesimal dos requeijões light adicionados de concentrado protéico do soro.

\begin{tabular}{|c|c|c|c|c|c|c|c|}
\hline & Umidade (\%) & Gordura (\%) & Proteína (\%) & Cinzas (\%) & Carboidratos (\%) & ESD (\%) & Caloria (Kcal) \\
\hline F1 & $64,9 \pm 0,1$ & $16,5 \pm 0,2$ & $14,0 \pm 0,3$ & $3,1 \pm 0,1$ & $1,4 \pm 0,1$ & $18,6 \pm 0,3$ & 210,3 \\
\hline $\mathrm{F} 2$ & $69,8 \pm 0,1$ & $16,4 \pm 0,1$ & $10,0 \pm 0,2$ & $2,7 \pm 0,2$ & $1,2 \pm 0,0$ & $13,9 \pm 0,0$ & 192,2 \\
\hline F3 & $69,8 \pm 0,1$ & $10,5 \pm 0,1$ & $14,4 \pm 0,3$ & $3,3 \pm 0,5$ & $2,1 \pm 0,7$ & $19,8 \pm 0,0$ & 165,0 \\
\hline $\mathrm{F} 4$ & $65,5 \pm 0,1$ & $11,5 \pm 0,3$ & $18,1 \pm 0,0$ & $3,2 \pm 0,1$ & $1,7 \pm 0,3$ & $23,0 \pm 0,2$ & 182,9 \\
\hline F5 & $71,0 \pm 0,1$ & $13,0 \pm 0,2$ & $12,1 \pm 0,3$ & $2,7 \pm 0,0$ & $1,3 \pm 0,0$ & $15,9 \pm 0,3$ & 170,3 \\
\hline F6 & $67,2 \pm 0,0$ & $18,5 \pm 0,5$ & $10,5 \pm 0,1$ & $2,5 \pm 0,0$ & $1,3 \pm 0,5$ & $14,3 \pm 0,5$ & 213,7 \\
\hline F7 & $63,5 \pm 0,1$ & $13,1 \pm 0,1$ & $18,5 \pm 0,3$ & $2,9 \pm 0,0$ & $1,9 \pm 0,6$ & $23,4 \pm 0,2$ & 199,7 \\
\hline F8 & $66,7 \pm 0,0$ & $8,5 \pm 0,1$ & $19,4 \pm 0,2$ & $3,4 \pm 0,3$ & $2,1 \pm 0,3$ & $24,9 \pm 0,2$ & 162,0 \\
\hline F9 R1 & $67,2 \pm 0,0$ & $13,6 \pm 0,2$ & $15,1 \pm 0,2$ & $2,8 \pm 0,0$ & $1,4 \pm 0,1$ & $19,3 \pm 0,2$ & 188,2 \\
\hline F9 R2 & $67,0 \pm 0,1$ & $13,8 \pm 0,2$ & $15,0 \pm 0,1$ & $2,9 \pm 0,1$ & $1,3 \pm 0,3$ & $19,2 \pm 0,3$ & 189,2 \\
\hline F9 R3 & $67,2 \pm 0,2$ & $13,2 \pm 0,2$ & $15,5 \pm 0,2$ & $2,8 \pm 0,1$ & $1,3 \pm 0,2$ & $19,6 \pm 0,3$ & 185,9 \\
\hline
\end{tabular}

F1: 17,3\%G e 65\%U; F2: 17,3\%G e 70\%U; F3: 10,2\%G e 70\%U; F4: 10,2\%G e 65\%U; F5: 13,7\%G e 71\%U; F6: $18,7 \% G$ e $67,5 \% U$; F7: 13,7\%G e 64\%U; F8: 8,7\%G e 67,5\%U; F9 (ponto central): 13,7\%G e 67,5\%U. \%ESD (extrato seco desengordurado): 100 - \% gordura - \% umidade. Desvio-padrão calculado com base nas replicatas. 
um aumento de $13,85 \%$ para $19,76 \%$ do ESD quando o teor de gordura foi reduzido de $16,4 \%$ para $10,5 \%$. O mesmo comportamento foi verificado para os requeijões F6, F9 e F8 (aproximadamente 67,5\%U) e também para as formulações F1 e F4 (65\%U). O conteúdo de umidade também provocou alterações no ESD dos requeijões, sendo que o ESD das formulações F1 e F2 aumentou de $13,85 \%$ para $18,06 \%$ quando o teor de umidade diminuiu de $69,75 \%$ para $64,90 \%$. O mesmo caso foi observado para as formulações F5, F9 e F7 (13,7\%G) e também para F3 e F4 (aproximadamente 10,2\%G). Os teores de gordura e de umidade influenciaram inversamente no percentual de ESD dos requeijões, sendo que produtos com combinações diferentes de gordura e umidade (F1, F3 e F9) apresentaram teor de ESD semelhantes (aproximadamente 19,3\%), devido à compensação da umidade no teor de ESD gerado pela redução de gordura.

Quanto ao valor calórico, o requeijão com menor teor de gordura combinado com o maior teor de umidade (F3-10,2\%G e70\%U) apresentou menor energia que as demais formulações, o que é muito interessante para a indústria, uma vez que este produto terá menor custo de produção (menor utilização de creme e de massa) e oferecerá baixa caloria. O valor calórico apresentado pelo requeijão F3 (165Kcal) foi inferior ao conteúdo energético encontrado por GALLINA et al. (2008) para requeijão cremoso, sendo verificado 210 Kcal.

Aceitabilidade sensorial dos requeijões light No Mapa de Preferência Interno, gerado para os dados de aceitação da textura dos requeijões (Figura 2), o primeiro componente principal explicou $54,22 \%$ e o segundo 21,72\%, totalizando, portanto, 75,94\% da variância entre as formulações. Assim, duas dimensões foram consideradas. A separação espacial dos requeijões sugere a formação de quatro grupos distintos, sendo um grupo formado pelos requeijões F1, F3 e F9, outro formado pelas formulações F4, F7 e F8, um terceiro grupo formado pelo requeijão F2 e um último grupo constituído pelas formulações F5 e F6.

Na figura 2, os consumidores estão representados por pontos em que cada abscissa e ordenada de um ponto é, respectivamente, a correlação linear entre o consumidor e os componentes principais. A correlação dos consumidores com pelo menos um dos componentes indica diferença na aceitação das formulações. Nesse tipo de representação gráfica, os consumidores se localizam próximos aos produtos que eles gostaram. Dessa forma, as formulações F1, F3 e F9 apresentaram maior aceitação quanto à textura, seguidos dos requeijões F5 e F6. Já os requeijões F2, F4, F7 e F8 não foram aceitos pelos consumidores em relação à textura. A não aceitação sensorial dos requeijões F4, F7 e F8 pode ser explicada devido ao elevado teor ESD (aproximadamente 24\%) desses produtos, os quais apresentaram uma matriz protéica muito rígida, desfavorecendo a textura dos requeijões. Para o requeijão F2, o inverso foi verificado, já que a elevação do teor de umidade a 69,8\%, combinado com $16,4 \%$ de gordura resultou em alta dissolução do ESD, conferindo uma textura muito mole ao requeijão, o que também prejudicou a aceitação do produto.

Verificou-se que os requeijões F1, F3 e F9, formulações com diferentes níveis de gordura,

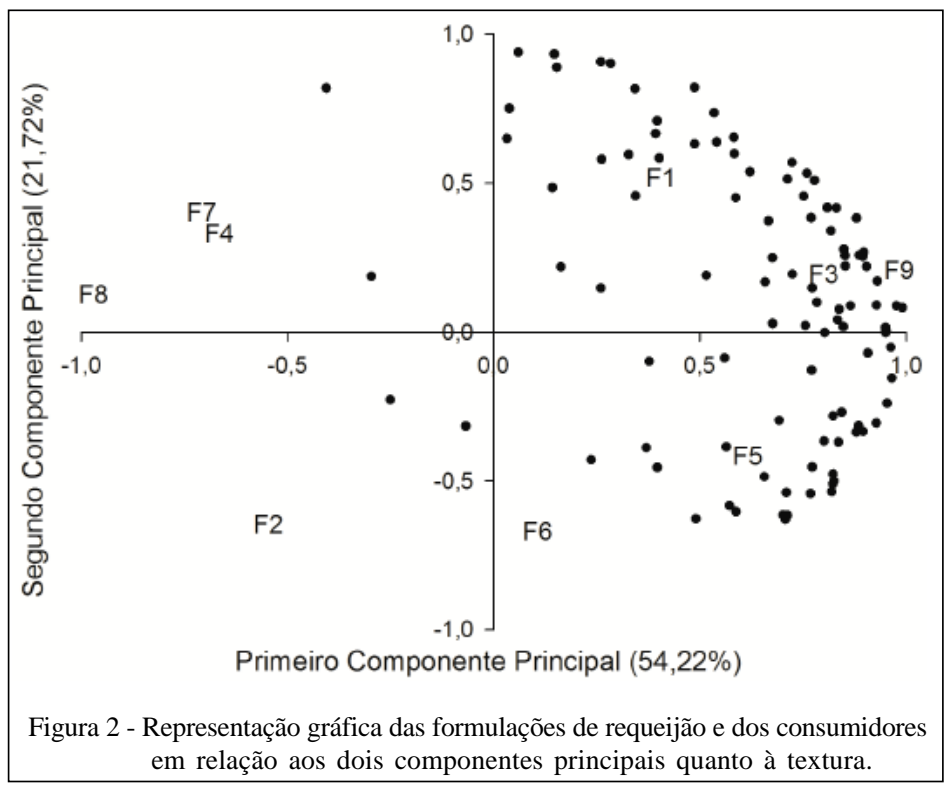

Ciência Rural, v.42, n.2, fev, 2012. 
obtiveram aceitação sensorial semelhante quando combinados com diferentes teores de umidade, ou seja, o requeijão com $17,3 \%$ de gordura e $65 \%$ de umidade (F1) apresentou aceitabilidade sensorial equivalente ao produto com $10,2 \%$ de gordura e $70 \%$ de umidade (F3) e, também, equivalente ao requeijão com 13,7\% de gordura e 67,5\% de umidade (F9). É importante ressaltar que essas formulações apresentaram teor de ESD muito próximos (aproximadamente 19,3\%) o que evidencia que a redução de gordura em requeijão deve ser aliada ao aumento no teor de umidade para que ocorra equilíbrio na textura do produto.

No Mapa de Preferência Interno para os dados de aceitação referente à aceitação da impressão global dos requeijões (Figura 3), os dois componentes principais juntos explicaram $72,5 \%$ da variância entre as formulações de requeijão. Como se pode observar, a separação espacial das formulações para a impressão global teve o mesmo comportamento quando comparado ao atributo textura, ou seja, os grupos sugeridos pela análise dos dados da impressão global são idênticos aos grupos sugeridos pela ACP dos dados de aceitação da textura e os consumidores se encontram localizados, na sua maioria, nos mesmos quadrantes. Este resultado sugere que a aceitabilidade sensorial dos requeijões foi definida principalmente pela textura dos produtos. Segundo RAPACCI et al. (1998), a textura é uma propriedade que tem papel fundamental na aceitação do requeijão cremoso e, em muitos casos, é tão importante para o consumidor quanto um atributo de sabor e aroma. Tal resultado evidencia a importância do estudo da relação do teor de gordura e de umidade e, consequentemente, do ESD na textura em requeijões light. Resultado semelhante foi encontrado por CUNHA et al. (2006) na avaliação da aceitabilidade de queijos com diferentes teores de gordura e umidade. Os autores verificaram que a textura foi determinante na aceitação global dos queijos e os produtos com ESD próximos de $23 \%$ foram mais aceitos frente ao produto com $30 \%$, indicando que o elevado conteúdo protéico prejudicou a textura do requeijão.

A relação existente entre o teor de ESD e a aceitabilidade sensorial da textura de requeijão light pode ser facilmente visualizada na figura 4 , a qual é uma representação gráfica da superfície de resposta da aceitabilidade em função do teor de gordura e umidade. Assim, é possível identificar diversas combinações entre gordura e umidade que atendam às expectativas dos consumidores em relação à textura dos requeijões. Quanto menor é o teor de gordura, maior deve ser o conteúdo de umidade do produto para que este apresente textura agradável. No entanto, esta correlação deve respeitar um valor de ESD em torno de 19-20\%. Tal informação é de grande valia para as indústrias, pois será possível oferecer uma diversidade de produtos que atendam às expectativas dos consumidores e ainda apresentem baixo custo de produção, além de estratégias de marketing que poderão ser realizadas devido à possibilidade de produzir um requeijão com baixo teor de gordura e com qualidade sensorial.

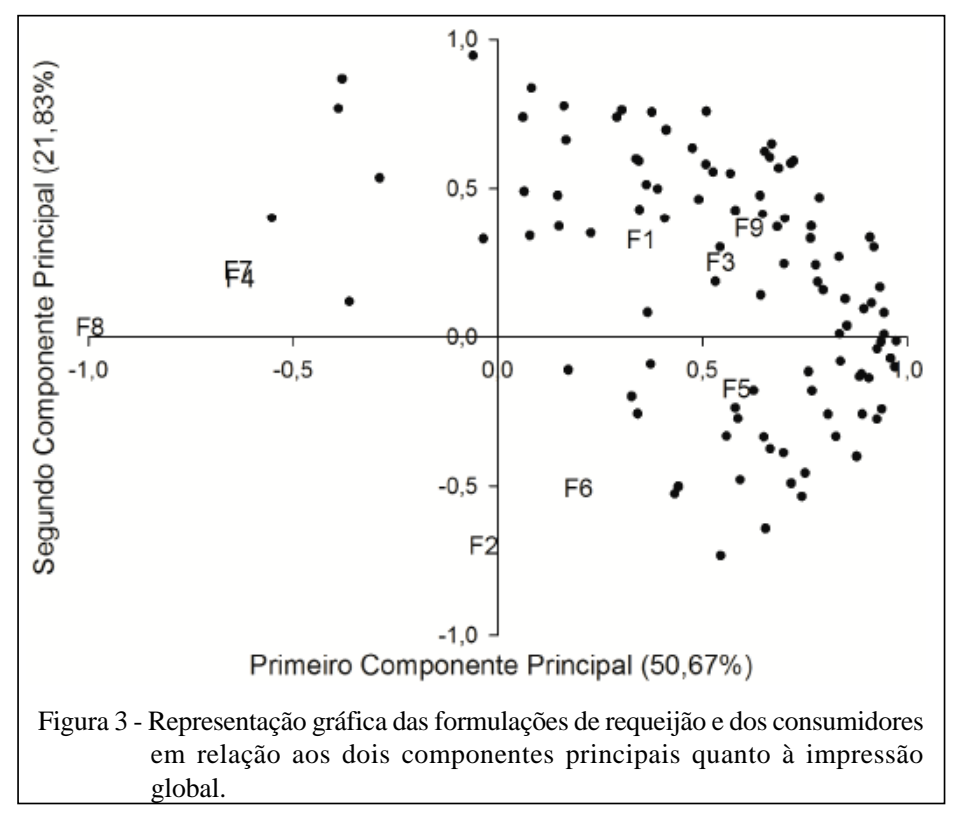

Ciência Rural, v.42, n.2, fev, 2012. 


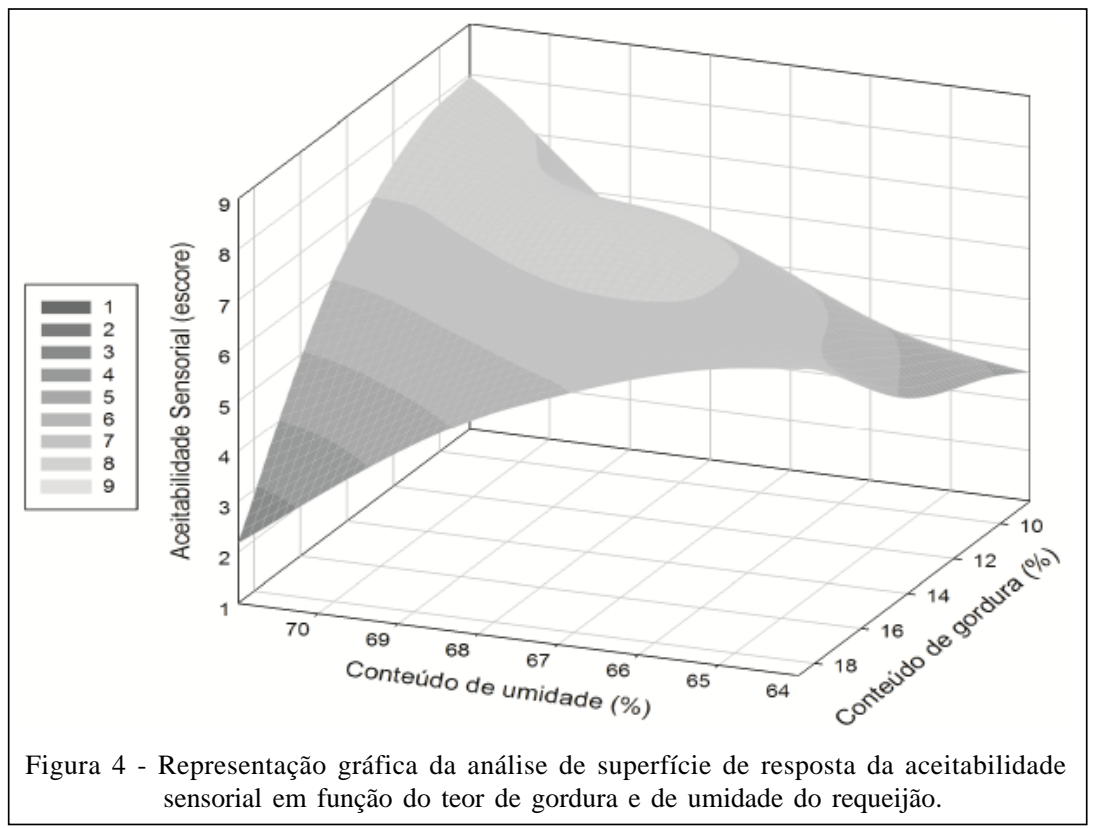

\section{CONCLUSÃO}

Os teores de gordura e de umidade se mostraram fatores determinantes na aceitabilidade sensorial da textura de requeijão light. Formulações com diferentes níveis de redução de gordura, aliadas ao aumento gradual no teor de umidade, apresentaram aceitação sensorial semelhantes, evidenciando que a aceitabilidade sensorial do requeijão cremoso light está diretamente relacionada com o equilíbrio no ESD. O equilíbrio gerado no ESD das formulações F1 (17,3\%G e 65\%U), F3 (10,2\%G e 70\%U) e F9 (13,7\%G e 67,5\%U) foi determinante na aceitabilidade sensorial dos requeijões, os quais foram os mais aceitos. Dentre as três formulações mais aceitas, o requeijão com menor teor de gordura combinado com o maior teor de umidade (F3) apresentou menor valor calórico que as demais formulações, o que é muito interessante para indústria, uma vez que este produto terá menor custo de produção, apresentará a mesma qualidade sensorial que as formulações com maior teor de gordura (F1 e F9) e ainda oferecerá menor conteúdo energético, além do apelo de marketing que poderá ser realizado devido a este requeijão apresentar uma redução de $65 \%$ do teor de gordura em relação ao produto tradicional. Assim, este estudo possibilita a obtenção de uma gama de produtos que atendam às expectativas dos consumidores.

\section{AGRADECIMENTOS}

Os autores agradecem ao Conselho de Desenvolvimento Científico e Tecnológico (CNPq) e à
Fundação de Amparo à Pesquisa do Estado de Minas Gerais (Fapemig) pelo apoio financeiro.

\section{REFERÊNCIAS}

ALVES, R.M.V. et al. Effect of light and packages on stability of spreadable processed cheese. International Dairy Journal, v.17, n.4, p.365-373, 2007. Disponível em: <http:// www.sciencedirect.com/science/journal/09586946/17/4>. Acesso em: 18 nov. 2011. doi: 10.1016/j.idairyj.2006.04.004.

BRASIL. Vigilância Sanitária, Ministério da Saúde (ANVISA). Portaria $\mathrm{n}^{\circ}$ 27, de 13 de Janeiro de 1998. Regulamento Técnico de Informação nutricional complementar. Diário Oficial da União, Brasília, DF, 1998.

BRASIL. Instituto Adolfo Lutz. Métodos físico-químicos para análise de alimentos. 4.ed. Brasília, 2005. 1018p.

CUNHA C.R. et al. Use of low concentration factor ultrafiltration retentates in reduced fat "Minas Frescal" cheese manufacture: effect on composition, proteolysis, viscoelastic properties and sensory acceptance. International Dairy Journal, v.16, n.3, p.215-224, 2006. Disponível em: <http:/ /www.sciencedirect.com/science/journal/09586946/16/3>. Acesso em: 18 nov. 2011. doi: 10.1016/j.idairyj.2005.03.004.

GALLINA, D.A. et al. Influence of storage temperature on the texture profile and colour characteristics of UHT requeijão cremoso. Brazilian Journal of Food Technology, v.11, p.169-174, 2008. Diposnível em: <http://bjft.ital.sp.gov.br/ index.php>. Acesso em: 18 nov. 2011.

HENNELLY, P.J. et al. Textural rheological, and microstructural properties of imitation cheese conitaing inulin. Journal of food engineering, v.75, n.3, p.388-395, 2006. Disponível em: <http:/ /www.sciencedirect.com/science/journal/02608774/77/3>. Acesso em: 18 nov. 2011. doi:10.1016/j.jfoodeng.2005.04.023. 
KAHYAOGLU, T.; KAYA, S. Effects of heat treatment and fat reduction on the rheological and functional properties of Gaziantep cheese. International Dairy Journal, v.13, p. 867-875, 2003. Disponível em: <http://www.sciencedirect.com/ science/journal/09586946/13/11>. Acesso em: 18 nov. 2011. doi: 10.1016/S0958-6946(03)00113-4.

METZGER, L.E. et al. Effect of milk preacidification on low fat Mozzarella cheese: ll. Chemical and functional properties during storage. Journal of Dairy Science, v.84, n.6, p.1348-1356, 2001. Disponível em: <http://www.journalofdairyscience.org>. Acesso em: 18 nov. 2011. doi: 10.3168/jds.10.3168/jds.S00220302(01)70166-X.

MINIM, V.P.R. Análise sensorial-estudo com consumidores. 2.ed. Viçosa: Universidade Federal de Viçosa, 2010. 308p.

PERRY, D.B. et al. Manufacture of low fat Mozzarella cheese using exopolysaccharide-producing starter cultures. Journal of Dairy Science, v.81, n.2, p.563-566, 1998. Disponível em: <http://www.journalofdairyscience.org>. Acesso em: 18 nov. 2011. doi: 10.3168/jds. S0022-0302(98)75608-5.

RAPACCI, M. et al. Requeijão cremoso: avaliação do perfil de textura. Revista Indústria de Laticínios, v.3, p.64-69, 1998. (Impresso)

ROMEIH, E.A. et al. Lowfat white-brined cheese made from bovine milk and two commercial fat mimetics: chemical, physical and sensory attributes. International Dairy Journal, v.12, n.6, p.525-540, 2002. Disponível em: <http:// www.sciencedirect.com/science/journal/09586946/12/6>. Acesso em: 18 nov. 2011. doi: 10.1016/S0958-6946(02)000432 .

RUDAN, M.A. et al. Effect of fat reduction on chemical composition, proteolysis, functionality, and yield of Mozzarella cheese. Journal of Dairy Science, v.82, n.4, p.661-672, 1999. Disponível em: http://www.journalofdairyscience.org. Acesso em: 18 nov. 2011. doi: 10.3168/jds.S0022-0302(99)75282-3.

SHEEHAN, J.J.; GUINEE, T.P. Effect of $\mathrm{pH}$ and calcium level on the biochemical, textural and functional properties of reducedfat Mozzarella cheese. International Dairy Journal, v.14, n.2, p.161-172, 2004. Disponível em: <http:// www.sciencedirect.com/science/journal/09586946/14/2>. Acesso em: 18 nov. 2011. doi: 10.1016/S0958-6946(03)00167-5.

SOARES, F.M. et al. Influência do concentrado protéico de soro na composição do requeijão em barra com teor reduzido de gordura. Arquivo Brasileiro de Medicina Veterinária e Zootecnia, v.54, p.1-7, 2002.

UNITED STATES DEPARTMENT OF AGRICULTURE. USDA. Composition of foods. Washington, 1963. 190p. 